\title{
Effectiveness of Isoniazid Preventive Therapy on Incidence of Tuberculosis in Adult People Living with HIV in Selected Districts of Rwanda
}

\author{
Marie C Uwamahoro ${ }^{1}$, Lonia M Mwape ${ }^{2}$, Soka Nyirenda ${ }^{2}$ \\ ${ }^{1}$ University of Rwanda, College of Medicine and Health Sciences, Rwanda \\ ${ }^{2}$ University of Zambia, School of Medicine, Zambia
}

\section{Background}

Tuberculosis is a major cause of death in people living with HIV. Despite available evidence suggesting that Isoniazid Preventive Therapy (IPT) reduces the incidence of tuberculosis among people living with HIV, its implementation is low. The present study intended to establish whether Isoniazid reduced the incidence of active tuberculosis in people living with HIV.

\section{Methods}

This was a retrospective cohort design using medical records of 2,172 people living with HIV (PLHIV) from six health facilities in three districts of Rwanda. An SPSS version 20 statistical analysis program was used to analyze the data. Survival analysis and Poisson regression were used to compare rates and factors contributing to TB in PLHIV on IPT and those not on IPT.

\section{Results}

The overall TB incidence rate (IR) was 1.131 cases per 100 person-years. The IR of TB in patients on IPT was significantly lower than those who were not on IPT (0.56/100PY vs 2.04/100PY) and Incidence Rate Ratio was 0.275 . Cox proportional hazard model revealed $81 \%$ reduction in TB risk among patients who received IPT. Being on anti retroviral therapy, having CD4 cell count $>350$, HIV clinical stage 1 and 2 and high income were factors contributing to lower IR of TB among people living with HIV on IPT. Patients on IPT lost their protection gradually but it was rapidly lost in patients not on IPT.

\section{Conclusion}

The use of IPT in people living with HIV was associated with a low IR of TB. The results would indicate a need to scale up the IPT to other health facilities.

Key words: isoniazid preventive therapy, tuberculosis, people living with HIV, Rwanda 